\title{
¿Cómo construir cuerpos individuales en el universo cartesiano?
}

\author{
¿How to build individual bodies in the \\ Cartesian universe? \\ Carlos Alberto CARdona SuÁrez ${ }^{1}$ \\ Universidad del Rosario (Bogotá-Colombia) \\ Juan Raúl LoAiza Arias \\ Universidad del Rosario (Bogotá-Colombia) \\ Humboldt-Universität zu Berlin (Alemania)
}

Recibido: 18/02/2015

Aceptado: 23/03/2015

\section{Resumen}

En el presente artículo se muestra que en la cosmología cartesiana no resulta claro cómo se puede hablar o referir a cuerpos individuales en el marco de un universo absolutamente compacto. Se defiende que el concepto de cuerpo individual en el interior de dicha cosmología se puede construir como una ficción del espíritu. Un cuerpo individual se puede construir como la extensión que se encuentra encerrada en una superficie maximal cuyas subdivisiones carecen de movimiento relativo entre sí. Se muestra, también, que la cosmología cartesiana puede presentarse como un modelo trivial de la Teoría $M$.

Palabras clave: cuerpo, vacío, individuación, movimiento, Teoría M.

\begin{abstract}
This article argues that in the Cartesian Cosmology it is not clear how we can talk about or refer to individual bodies in the context of a compact universe. It defends that the concept of individual body inside of this Cosmology can be built as a fiction of the spirit. This concept can be constructed as the extension that is locked up in a

\footnotetext{
${ }^{1}$ Artículo escrito en el marco del proyecto de investigación La pirámide visual: evolución de un instrumento conceptual apoyado por el Fondo de Investigaciones de la Universidad del Rosario.
} 
maximal surface whose subdivisions have no relative movement between them. It is also shown that Cartesian Cosmology can be seen as a trivial model of M-theory.

Keywords: body, vacuum, individuation, movement, M-theory.

\section{Introducción}

Incómodo con la proliferación de formas substanciales como último recurso para explicar la compleja diversidad de fenómenos naturales, Descartes decidió inaugurar un ambicioso programa minimalista de investigación, programa orientado a explicar la totalidad de dichos fenómenos a partir del menor número de elementos que el espíritu pudiese aprehender con tal claridad y distinción que resultaría ininteligible negarse a reconocer dichos elementos. Descartes llegó a convencerse de que la extensión atribuida a los objetos físicos podía ser ese elemento. No obstante, la extensión por sí sola no basta, es decir, la extensión es un elemento necesario, pero no es suficiente. La gran diversidad que podemos observar en el mundo tendría que contemplarse a la manera de diferentes modalidades en una única substancia de base (lo extenso). Estas modalidades básicas de la substancia extensa se reducen a figura (cuando contamos con algún criterio para delimitar un objeto) y movimiento. Así las cosas, el programa mecanicista de Descartes aspira a explicar todos los acontecimientos del mundo físico presuponiendo tan sólo una substancia extensa a la cual se le puede atribuir figura (límites individuales) y movimiento como modalidades. Agregar algún elemento adicional es traicionar las exigencias minimalistas del programa.

En el programa científico de Descartes se siente palpitar una profunda tensión entre dos polos que no son fácilmente conciliables. En algunos pasajes el filósofo da muestras de advertir la incomodidad. Sin embargo, en el horizonte de la obra completa, la tensión se ignora o se hace a un lado como si se tratara de una deficiencia menor. A nuestro juicio, dicha tensión, que procederemos a explicar a continuación, no puede ignorarse y exige, más bien, una cuidadosa atención si se quiere mantener firme el núcleo del programa de investigación.

Primer polo de la tensión. Descartes sostiene que el único atributo que necesitamos adscribirle a los objetos físicos es la extensión. En las Reglas para la dirección del espiritu estipula que «Entendemos por extensión todo aquello que tiene longitud, latitud y profundidad, sin indagar si es un cuerpo verdadero o sólo espacio». ${ }^{2} \mathrm{De}$ allí, como una posibilidad, se desprende que podemos hablar de la existencia de

\footnotetext{
${ }^{2}$ R. Descartes, (1897-1910). Oeuvres de Descartes. Paris: Léopold Cerf (editor); publicadas por Charles Adam \& Paul Tannery con el auspicio del Ministerio de Instrucción Pública; 12 volúmenes; en adelante, antecedida del título de la obra, usaremos la sigla $A T$ para remitir a dicha edición, Reglas, $A T$, X, p. 442, Regla XIV.
} 
fragmentos extensos en el espacio sin la necesidad de imaginarlos ocupados por cuerpo alguno. Sin embargo, en los Principios de la filosofía Descartes critica a quienes pretenden que se pueda hablar de espacios vacíos separados de la substancia que contienen: «a partir de que un cuerpo es extenso en longitud, anchura y profundidad, tenemos razón para concluir que es substancia [...] debemos concluir lo mismo del espacio que se supone vacio: a saber, que dado que en él hay extensión, necesariamente hay en él substancia». ${ }^{3}$ Nuestro razonamiento, cree el filósofo, pretende ocuparse de algo ininteligible cuando aduce que existen fragmentos extensos y vacíos a la vez. Descartes también demuestra, a modo de corolarios, que los cuerpos físicos no contienen átomos indivisibles, ${ }^{4}$ y que la extensión del mundo es indefini$\mathrm{da}^{5}$. Las demostraciones cartesianas sugieren que el universo así concebido es compacto en todas sus dimensiones por cuanto no se reconocen intersticios vacíos que pudiesen separar una porción de materia de otra. ${ }^{6}$ En ese orden de ideas, y teniendo en cuenta que no contamos con formas substanciales o con conceptos que pudiesen hacer las veces de dichas formas, hemos de concluir que no contamos con criterios que permitan distinguir con claridad un objeto físico de otro. Todo el universo es un plenum o un continuo que no admite diferenciación especial entre un sector u otro. Así entonces, el programa minimalista de Descartes no ofrece, prima facie, criterios de individuación de objetos.

Segundo polo de la tensión. Si bien no contamos con criterios de individuación de objetos, las leyes fundamentales que Descartes postula para su universo hacen referencia a objetos individuados en el marco (o trasfondo) del plenum cartesiano. En la Segunda Parte de los Principios de la filosofía sostiene Descartes que Dios es la única causa general de cualquier movimiento que se da en el universo y, por lo tanto, Dios es el origen primigenio de cualquier diversidad que pretendamos adscribir al plenum. También reconoce el filósofo que Dios obra de una manera tal que no admite variación alguna; en ese orden de ideas, cada vez que observamos en el mundo una aparente modificación, debemos estar en condiciones de hallar en el trasfondo el sello de la invariabilidad de la obra de Dios. De lo anterior se sigue que los aparentes cambios en el plenum deben seguir las mismas leyes ${ }^{7}$ que habrían de fundamentarse en la invariabilidad de Dios $^{8}$.

\footnotetext{
${ }^{3}$ Principios (edic. francesa), AT, IX, parte II, $\S 16$, pp. 71-72.

${ }^{4}$ Principios (edic. francesa), $A T$, IX, parte II, $\$ 20$, p. 74.

${ }^{5}$ Principios (edic. francesa), AT, IX, parte II, $\$ 21$, p. 74.

${ }^{6}$ Según Lewis, sólo el universo como un todo constituye una substancia extensa genuina. Cfr. G. Lewis, L'individualité selon Descartes, 1950, Paris: J. Vrin, p.p. 39-41.

${ }^{7}$ Estas leyes se formularon con precisión en la construcción de la fábula del mundo que el autor concibió en los capítulos VI y VII de Le monde, Traité de la lumiere (AT, XI). En los Principios de la filosofía se volvieron a presentar dichas leyes como si pudieran derivarse de una cierta fundamentación metafísica.

${ }^{8}$ Principios (edic. francesa), AT, IX, parte II, § 36. pp. 83-84.
} 
El problema que suscitan estas leyes de la naturaleza reside en que ellas aluden a lo que habría de ocurrirle a cuerpos individuados, en circunstancias en las que se reconoce la intervención de otros cuerpos igualmente individuados. Sigamos con atención la formulación que de cada ley se hace en los Principios, subrayaremos la alusión que se hace a cuerpos diferenciados en el plenum cartesiano. La primera ley reza así: «cada cosa permanece en el estado en el que está mientras que nada modifica ese estado». ${ }^{9}$ Si la única cosa que existe, como mostraremos más adelante, es el plenum cartesiano, dicha ley afirma que el universo en todo su conjunto permanece inalterable salvo que Dios, que es el único que podría intervenir, decida provocar alguna alteración local. Si la expresión "cada cosa" alude a cuerpos diferenciados, o mejor, porciones del plenum, hemos de protestar porque aún no sabríamos a qué reconocer como un cuerpo individuado. Descartes ofrece en la defensa de dicha ley ejemplos que aluden al reconocimiento de cuerpos separados con los que tenemos ya cierta familiaridad en nuestro devenir cotidiano y recalca en forma categórica el sentido de su ley: «cada cosa en particular se mantiene en el mismo estado en tanto que es posible y sólo lo modifica en razón del encuentro con otras». ${ }^{10}$ En la versión de este pasaje en la edición latina se precisa mejor a qué se refiere con "cada cosa en particular", se dice allí «quate nus est simplex et indivisa». ${ }^{11}$ Sin embargo, no sabríamos qué la hace simple y, de otro lado, hemos dicho que nada en el mundo físico es por principio indivisible.

La segunda ley dice: «Todo cuerpo que se mueve tiende a continuar su movimiento en línea recta». ${ }^{12} \mathrm{El}$ enunciado alude claramente a la posibilidad de diferenciar un cuerpo del resto que le acompaña. Sin embargo, la metafísica cartesiana ni nos dice cómo separar cuerpos individuales del plenum, ni admite que los cuerpos como unidades independientes nos sean dados ab initio. Si la ley alude a lo que ha de ocurrirle al universo en su conjunto, no sabríamos qué sentido o significado darle a "movimiento en línea recta" pues no tendríamos argumentos para presuponer la existencia de un espacio absoluto más amplio en el que estuviera contenido el universo físico cartesiano y en el que dicho universo pudiese moverse en línea recta sin que nadie que lo habita pudiese cerciorarse de ello.

Finalmente, la tercera ley propone: «si un cuerpo en movimiento choca con otro más fuerte que él, no pierde nada de su movimiento; ahora bien si encuentra otro más débil y que puede mover, pierde tanto movimiento como comunica al otro».. ${ }^{13}$ Esta ley (conservación de la cantidad de movimiento) dice qué es lo que habría de ocurrir si dos cuerpos se estrellan. De hecho se presupone la existencia de al menos

\footnotetext{
${ }^{9}$ Principios (edic. francesa), AT, IX, parte II, $\S 37$, p. 84, el resaltado es nuestro.

${ }^{10}$ Principios (edic. francesa), $A T$, IX, parte II, $\S 37$, p. 84.

11 "En cuanto es simple e indivisa"; Principios (edic. latina), AT, VIII, parte II, §37, p. 62.

${ }^{12}$ Principios (edic. francesa), AT, IX, parte II, $\S 39$, p. 85.

${ }^{13}$ Principios (edic. francesa), AT, IX, parte II, $\S 40$ p. 86; el subrayado es nuestro.
} 
dos cuerpos diferenciados en el mundo. Esta ley supone que estamos en condiciones de distinguir un cuerpo del otro y advertir qué tendría que ocurrir con ellos después del encuentro, siempre que podamos garantizar que conservan la unidad que debemos presuponer al comienzo. La ley dice que si dos cuerpos que se reconocen diferentes se estrellan, ellos -siempre que no pierdan su unidad después del encuentro- intercambian cantidades de movimiento.

En resumen: el universo fingido por Descartes no admite intersticios vacíos, por lo tanto todo él es un plenum compacto. No obstante, las leyes de la naturaleza hacen referencia a cuerpos separados del resto del continuo; de hecho a cuerpos particulares. Pretendemos mostrar que se puede salvar la tensión sugerida si admitimos que la referencia a cuerpos individuales alude a una construcción en la que están implícitas algunas operaciones ficticias agenciadas por el espíritu (por la imaginación realmente). En particular, un cuerpo es aquella porción del continuo que queda encerrada en una superficie maximal que satisface ciertas condiciones que se harán explícitas en el artículo. De otra parte, mostraremos que resulta útil ver la cosmología cartesiana como un modelo trivial para la teoría ontológica abstracta propuesta por Michael Jubien; este movimiento nos llevará a formular una versión más sofisticada de superficie maximal. Finalmente dejaremos un espacio para comentar muy brevemente algunas consecuencias inmediatas de la propuesta.

\section{Construcción de cuerpos como ficciones del espíritu}

El universo de Descartes se parece, pues, a una masa compacta de gelatina. Una masa sin intersticios. No hay lugar para rincones vacíos, cualquier perturbación en un extremo tiene que sentirse inmediatamente en todo el universo ${ }^{14}$ de tal manera que en el acto se frustra cualquier pretensión de ver nacer un lugar vacío. Hay dos dificultades que inmediatamente surgen: (i) ¿cómo podemos individuar pretendidos objetos particulares si no hay criterios geométricos (o físicos) que permitan aislar una parte del resto de la gelatina?; (ii) ¿cómo definir el movimiento si no podemos decir que es la simple transición de un cuerpo físico desde un lugar hasta otro, toda vez que el espacio en la cosmología cartesiana no se puede definir con total independencia de los cuerpos físicos? Las leyes de la naturaleza hablan de lo que ha de ocurrirle a una parte de la materia cuando nadie interviene sobre ella, o de lo que le pasa a un cuerpo cuando se encuentra con otro; sin embargo no sabemos cómo separar una parte del resto o cómo hemos de identificar un cuerpo si todo el universo es compacto. Cuando Descartes presenta las condiciones del mundo que pretende fingir ${ }^{15}$ le dice enfáticamente al lector: «Suponemos además que Dios la divide [la materia]

\footnotetext{
${ }^{14}$ La comparación con una masa de gelatina no es del todo conveniente, pues dada la elasticidad de la gelatina, no es cierto que una perturbación en un lugar se haga sentir al instante en cualquier sector.

${ }^{15}$ Hacemos alusión a la fábula del mundo introducida en De la Luz.
} 
verdaderamente en numerosas partes, las unas mayores, las otras menores; las unas de una figura, las otras de otra, de cualquier tipo que nos plazca fingir. Ahora bien, todo ello sin que Dios separe unas de otras con el vacio entre sì». ${ }^{16}$ Un cuerpo no se distingue del otro por el vacío que media entre ellos. Si todo es una unidad compacta, ¿con qué derecho hablamos de cuerpos o partes diferenciadas? ¿Tienen los denominados cuerpos las formas (figuras) que se nos antoja fingir?

En los Principios de la filosofia, Descartes quiso distinguir entre, por un lado, lugar interior y lugar externo, y, por otro lado, entre movimiento según el uso común y movimiento propiamente dicho. Veamos si estas distinciones ayudan a disipar las dificultades; nos detendremos por separado en cada una de ellas. La distinción entre cuerpo y lugar interno es sólo una distinción de razón: así como no puedo concebir un cuerpo sin espacio interno, tampoco puedo concebir el segundo sin el primero. $\mathrm{Al}$ respecto señala el filósofo:

La diferencia que existe entre ellos sólo reside en que nosotros atribuimos al cuerpo una determinada extensión, que entendemos que cambia de lugar con él todas y cuantas veces el cuerpo es transportado; por otra parte, atribuimos al espacio una unidad tan general y tan vaga que después de haber retirado de un cierto espacio el cuerpo que lo ocupaba, no pensamos haber transportado también la extensión de este espacio, ya que nos parece que la misma extensión permanece alli..$^{17}$

Lugar interno y lugar externo se dicen de cuerpos individuados. Ahora bien, si la extensión (lugar interno) que es encerrada por un cuerpo cambia de posición de la misma manera como el cuerpo es transportado de aquí a allá, nos sentimos inclinados a pensar que es el movimiento al unísono de cada una de las partes encerradas lo que determina la unidad corporal. La unidad del cuerpo es, pues, la unidad que determina que cada una de sus partes se mueva de la misma manera que las demás, de suerte que cada una de ellas parece reposar con respecto a las otras. Es en ese sentido que cada cosa individual nos parece simple e indivisa. Decimos que una parte de la materia se mueve con respecto a otra si cambia con el tiempo la distancia que las separa (o la disposición, si pensamos en un movimiento circular). Lo anterior vale si podemos tener una idea o noción de distancia que sea anterior a la distinción de los cuerpos; supondremos que ese es el caso. Se dice que una cosa está en un lugar cuando está situada de una forma determinada con respecto a alguna otra cosa. ${ }^{18}$ El lugar externo es, de otra parte, la superficie que rodea inmediatamente la cosa y que no forma parte de ella sino del límite entre el cuerpo rodeado y el cuerpo que rodea. ${ }^{19}$

\footnotetext{
${ }^{16}$ De la Luz, AT, XI, p 34.

${ }^{17}$ Principios (edic. francesa), AT, IX, parte II, § 10, p. 68.

${ }^{18}$ Principios (edic. francesa), AT, IX, parte II, § 14, p. 71.

${ }^{19}$ El lugar externo evoca la caracterización aristotélica de lugar. Señala Aristóteles: «el lugar es lo que
} 
Así las cosas, si imaginamos una superficie ficticia (lugar externo) que envuelve un candidato a ser considerado como un cuerpo y advertimos que esta superficie carece de movimiento relativo con respecto a la unidad encerrada, podemos animarnos a considerar esta rebanada envolvente como si hiciese parte del cuerpo que estamos componiendo. En ese orden de ideas, la superficie no sería aún el lugar externo del pretendido cuerpo. A continuación podemos tomar la superficie que envuelve al cuerpo original aumentado con la superficie fallida para preguntar ahora si esta nueva superficie reposa o se mueve con respecto a la unidad del nuevo cuerpo examinado. Cuando encontremos en este proceso iterado una superficie que se mueve con respecto a la unidad encerrada, asumiremos que hemos llegado al límite determinante del cuerpo así construido. Esta última superficie se erige con todo derecho como el lugar externo. Este proceso que estamos imaginando debe concebirse sin dar lugar a modificaciones intermedias; pues bien puede ocurrir que entre un ensayo y el siguiente las partes involucradas cambien las posiciones relativas en virtud de sus interacciones.

Podemos imaginar el asunto de la siguiente manera: determinamos arbitrariamente una superficie que encierra por completo una porción pequeña de materia que podría llegar a ser considerada, prima facie, un cuerpo. Si, por un lado, notamos que fragmentos internos tienen entre sí movimiento relativo, procedemos a reducir la superficie inicial hasta conseguir que entre sus partes internas no exista movimiento relativo alguno. $\mathrm{Si}$, de otro lado, advertimos que las partes que constituyen la superficie reposan con respecto a las internas y estas carecen de movimiento relativo entre sí, nos animamos a ampliar las dimensiones de la parte encerrada. Así, seguiremos ampliando las dimensiones de la superficie, rebanada tras rebanada, hasta advertir que las partes de ella se mueven (cambian sus relaciones de distancia) con respecto al cuerpo encerrado. Esta superficie es, entonces, una superficie maximal. En otras palabras: si pretendemos aumentar dicha superficie, nos veríamos obligados a incluir fragmentos que tenemos la obligación de considerar en movimiento con respecto a las porciones encerradas por la superficie; si intentamos reducir la superficie, notaremos que ella deja por fuera fragmentos que aún se conservan en reposo con respecto a las partes encerradas por la misma. Esta superficie, que en algún sentido define los límites que encierran a un cuerpo, no debe entenderse como parte del cuerpo rodeado, sino solamente como el límite entre el cuerpo que rodea y el que es rodeado. El fragmento de universo así aislado puede considerarse como una unidad a la que denominamos cuerpo. Estos cuerpos pueden o bien gozar de una unidad que se mantiene por ciertos períodos de tiempo, o bien tener una dinámica que les permite ampliar o reducir las dimensiones con el paso del tiempo. Por esa razón es importante imaginar que el tiempo que nos toma hallar una superficie maximal sea reducido. En estas condiciones, un cuerpo no es una unidad que existe per se y que es anterior a cualquier determinación que pretendamos darle; un cuerpo es una

primariamente contiene aquello de lo cual es lugar, y no es una parte de la cosa contenida» (Física, IV, 211a). 
ficción o creación del intelecto. Somos nosotros quienes introducimos las superficies maximales que encierran aquellas porciones del universo que, en un momento, consideramos una unidad por cuanto, primero, no detectamos movimiento en su interior $y$, segundo, sí reconocemos variaciones en relación con las partes circundantes. Si asumimos que el universo es una masa compacta que no admite intersticios vacíos, este nos aparece como el continuo pleno en todo el sentido de la palabra. Ahora bien, la partición de los cuerpos que imaginamos en el universo es el resultado de una sofisticada ficción. Bien podemos decir que los cuerpos son disecciones-ficticias-de-lo-continuo.

¿Hasta dónde estamos autorizados a pensar que esta descripción hace justicia con lo que Descartes pudo tener en mente? Si nuestra respuesta es afirmativa, Descartes estaría más cerca de las tesis de un realista interno que lo que nos gustaría reconocer en principio. La siguiente aclaración de Descartes le da fuerza a nuestra interpretación que asume que la distinción de los cuerpos presentes en el universo cartesiano es una ficción del espíritu. Recuerda primero el autor que el universo está formado por una sola materia, que dicha materia se podría dividir sin límite alguno y que se puede fragmentar en partes que se mueven de modo diverso:

No hemos podido determinar de igual forma las dimensiones de las partes en las
que la materia está dividida, ni cuál es la velocidad con que tales partes se mueven,
ni cuáles son los círculos que describen al moverse. No ha sido posible esta determi-
nación, pues habiendo podido ser ordenadas por Dios en una infinidad de distintas
formas, sólo la experiencia y en modo alguno la fuerza del razonamiento, permite
conocer cuál de todas estas formas ha sido la elegida. Esta es la razón en virtud de
la cual y libremente podemos elegir aquella que deseemos siempre y cuando todo lo
que sea deducido sea enteramente acorde con la experiencia. ${ }^{20}$

También aclara el filósofo en Los principios: «en el caso de que exista [la substancia corpórea], cada parte de la misma que podamos determinar con nuestro pensamiento debe ser realmente distinta de las otras partes de esa substancia». ${ }^{21} \mathrm{En}$ la sexta meditación Descartes aclara que mientras, por un lado, no se pueden identificar partes en la sustancia pensante, toda vez que es el espíritu enteramente el que se ocupa ora de querer, ora de sentir, ora de concebir, por otro lado, sí es posible fragmentar en partes la substancia corpórea y ésta es una facultad que se reserva el espíritu. Aclara Descartes que en contraste con el espíritu: «no hay ninguna [cosa corporal] que yo no separe fácilmente en pedazos con mi pensamiento, que mi espiritu no divida fácilmente en muchas partes, y que, por consiguiente, yo no conozca que es divisible». ${ }^{22}$

\footnotetext{
${ }^{20}$ Principios (edic. francesa), AT, IX, parte III, § 46, p. 124.

${ }^{21}$ Principios (edic. francesa), AT, IX-2, parte I, § 60, p. 51.

${ }^{22}$ Meditaciones, $A T$, IX, medit. VI, p. 68.
} 
Apartes como los anteriores nos llevan a pensar que Descartes tenía en mente la posibilidad de divisar las fragmentaciones del plenum como ficciones del espíritu. Al elucidar la conexión entre los objetos físicos y matemáticos en la cosmología cartesiana, Gaukroger (1980) sostiene que es la actividad del intelecto la que, aplicándose a la imaginación, conecta los objetos de la matemática con los de la física. Los objetos de la matemática, en tanto objetos abstractos, son objetos indeterminados, mientras que los objetos de la física, en tanto parte del mundo de los cuerpos, son determinados. Dado que no podemos representarnos lo indeterminado como parte del mundo corpóreo, es el intelecto el que crea una ficción que, aplicada a la imaginación, representa los objetos indeterminados como determinados, las magnitudes generales como específicas, y, podríamos agregar, el plenum continuo como un conjunto de cuerpos individuados. ${ }^{23}$

Parte de la dificultad para individuar cuerpos deviene de la falta de una definición precisa de substancia en la obra de Descartes. De un lado, en el contexto metafísico la substancia puede definirse como aquella entidad que puede existir sin la ayuda de cosa alguna creada ${ }^{24}$ de otro, puede definirse en el contexto de la predicación como aquello que es sujeto de cualidades. Descartes se mueve de una acepción a otra sin percatarse de las dificultades que provoca. Así, por ejemplo, en la meditación tercera sostiene: «cuando pienso que la piedra es substancia [...] y así mismo que yo soy substancia»; $; 25$ esto nos lleva a reconocer una piedra como muestra particular de una substancia: un algo que se sabe independiente (e individualizado) de todo lo que le rodea. Sin embargo, del hecho de que una piedra pueda fragmentarse por la intervención de un agente físico (diferente a Dios) y pueda recomponerse después por la intervención de un agente humano que reúne nuevamente sus fragmentos, se infiere que a la piedra no le viene bien la primera acepción de substancia. Cuando nos valemos de la introspección para evaluar nuestros estados de conciencia que parecen remitir a la supuesta existencia de objetos externos, descubrimos que ellos remiten en forma esencial a algo que es extenso. La substancia (corpórea o pensante) es ese algo, en principio indeterminado, de lo que predicamos ora extensión, en el primer caso, ora pensamiento en el segundo. Al final de las respuestas a las segundas objeciones recogidas por el padre Mersenne, Descartes ofreció una prueba de la existencia de Dios siguiendo el método de la síntesis empleado por los geómetras. En el transcurso de dicha prueba presentó la siguiente definición:

Toda cosa en la cual, como en su sujeto, está insito algo, o sea, por la cual existe algo que concebimos, es decir, alguna propiedad, cualidad o atributo del que tenemos en nosotros una idea real, es llamada Substancia. Pues la sola idea precisa

\footnotetext{
${ }^{23}$ Cfr. S. Gaukroger, "Descartes' Project for a Mathematical Physics", en S. Gaukroger (editor), Descartes: Philosophy, Mathematics and Physics, Sussex: The Harvesters Press Ltd, 1980, pp. 109-110. ${ }^{24}$ Meditaciones, AT, VII, p. 14; Principios (edic. francesa), AT, IX, parte I, § 52, p. 47.

${ }^{25}$ Meditaciones, $A T$, VII, medit. III, p. 44.
} 
de substancia que tenemos es la de que se trata de una cosa en la cual existe formalmente o eminentemente lo que concebimos, o sea, lo que está objetivamente en alguna de nuestras ideas, pues la luz natural nos enseña que la nada no puede tener atributo alguno real. ${ }^{26}$

Explica después Descartes que la substancia en la que se encuentra ínsito el pensamiento ha de llamarse Espíritu y la substancia que ha de exigirse como sujeto inmediato de la extensión se llama Cuerpo. Así las cosas, nosotros concebimos las substancias a través de sus atributos e inferimos su presencia necesaria a partir de la luz natural que exige que los atributos lo han de ser de un sujeto específico. En Los principios se define atributo como aquel modo o cualidad que no se puede considerar de otra manera más que como dependiente de la substancia. ${ }^{27}$ Spinoza, influenciado por el espíritu cartesiano e imitando el more geométrico, presenta en su Ética una serie de definiciones a partir de las cuales infiere las consecuencias básicas de su filosofía. Entre las definiciones sugiere que substancia es aquello que es en sí y se concibe por sí (aquello cuyo concepto no precisa del concepto de otra cosa), atributo es aquello que el entendimiento percibe de una substancia como constitutivo de su esencia y modo es aquello que es en otra cosa (en una substancia). En la proposición 5 de su tratado demuestra que no puede haber dos o más substancias de la misma naturaleza. ${ }^{28}$ De hecho, si las pretendidas dos substancias se pudiesen diferenciar por el atributo, serían entonces dos substancias de naturaleza diferente; y si las pretendidas substancias se pudiesen diferenciar por sus afecciones (modos), dado que la substancia debe ser anterior a sus afecciones (proposición 1), al margen de las afecciones que las distinguen no habría dos substancias diferentes si pedimos para ellas identidad de naturaleza. Así las cosas, siempre que nos restrinjamos a las demandas de la filosofía cartesiana, no hay dos substancias que siendo diferentes compartan la naturaleza de la extensión.

En una carta enviada al padre Gibieuf (enero 19 de 1642) señala Descartes que la distinción entre substancia y sus atributos esenciales es sólo el resultado de una abstracción llevada a cabo por el intelecto. En el desarrollo de dicha aclaración, Descartes acude a las distinciones que trazamos entre sustancia extensa y la figura que la encierra. Señala el filósofo:

Cuando yo considero una figura sin pensar en la sustancia o extensión, estoy haciendo una abstracción mental. Puedo reconocer fácilmente esta abstracción después de considerar si yo en alguna forma he derivado la idea que tengo sólo a partir de la figura, sin considerar alguna otra idea más amplia que yo también encuentro

\footnotetext{
${ }^{26}$ AT, VII, p. 161.

${ }^{27}$ Principios (edic. francesa), AT, IX, parte I, § 56, p. 49.

${ }^{28}$ Cfr. B. Spinoza. Ética demostrada según el orden geométrico. Barcelona: Ediciones Orbis, 1980, pp. 47-50.
} 
en mí, a la cual ésta está unida en una forma tal que, aunque nosotros podamos pensar en una sin poner atención a la otra, nosotros somos no obstante incapaces de negar la una sin la otra cuando pensamos en las dos ideas juntas. Así, yo veo claramente que la ideas de una figura está así unida a la idea de extensión y así de substancia, dado que es imposible que yo conciba una figura mientras niegue que ésta tiene una extensión, o de una extensión mientras niegue que se trata de la extensión de una substancia. ${ }^{29}$

En ese orden de ideas, sólo existe una substancia extensa: la masa completa y compacta que constituye el universo físico en su totalidad. Cuando pretendemos valernos del término substancia para remitirnos a un objeto individualizado (una piedra, por ejemplo) es porque usamos de manera laxa la definición dado que podemos señalar una serie de afecciones o modos que determinan la diferencia con otros semejantes. Esto es lo que ocurre cuando concebimos una superficie maximal que, a la manera de una ficción del espíritu, encierra fragmentos que carecen de movimiento relativo entre sí y define el límite con otras partes que poseen movimiento relativo con respecto a los fragmentos encerrados. Cuando decimos que una piedra es un ejemplar de substancia extensa (en el sentido laxo) es porque por alguna razón nos hemos inclinado a crear una partición ficticia en ese pleno continuo que constituye la substancia extensa en su completitud; se trata de una partición ficticia que nos hace pensar que cada uno de los fragmentos de la piedra se mueve al unísono con los restantes cuando ella se desplaza desde aquí hasta allí.

La sugerencia de definir un cuerpo como la partición que crea el espíritu de una porción de material extenso encerrada en una superficie maximal de tal manera que sus fracciones carecen de movimiento relativo puede abrir el espacio para hablar de cuerpos particulares en las leyes físicas sin presuponer que ellos nos sean dados de antemano y sin renunciar al plenum. La fuerza de la definición aquí propuesta no descansa en el hecho o la probabilidad de que Descartes finalmente la viera con buenos ojos. No obstante, hemos mostrado que hay pasajes en la obra del filósofo que sugieren cierta empatía con la formulación.

Ocupémonos ahora de la distinción que Descartes había señalado en Los principios entre movimiento en sentido laxo y movimiento en sentido estricto. El movimiento según el uso común es la acción por la cual un cuerpo se traslada de un lugar a otro. Ahora, dado que lugar se puede entender en sentido interno y en sentido externo, la anterior definición o resulta ininteligible (si pensamos en el lugar interno) o requiere de una precisión mayor si alude al lugar externo. El movimiento en el sentido propio es: «la traslación de una parte de la materia o de un cuerpo de la vecindad de los que contactan inmediatamente con él y que

${ }^{29}$ AT, III, p. 475. 
consideramos como en reposo en la vecindad de otros $\gg .^{30}$ En ese orden de ideas y como habíamos señalado antes, es la diferencia de movimiento entre las partes que comparten una superficie limitante la que permite individuar partes de la materia para considerarlas bajo la unidad de un cuerpo. Descartes define así el concepto: «Entiendo por un cuerpo o bien por una parte de la materia todo lo que es transportado a la vez, aunque esté compuesto de partes diversas que emplean su agitación para producir otros movimientos». ${ }^{31} \mathrm{Si}$ la propuesta que hemos formulado es correcta, admitiremos, entonces, que es el entendimiento (la imaginación) quien finge la noción de un cuerpo particular para hacer distinciones que le permitan concebir la dinámica del mundo en una forma integrada. La construcción ficticia atiende al reconocimiento de movimientos relativos con respecto a la superficie circundante. De hecho, es la introducción fingida de cuerpos (o partes de la materia) la que permite introducir las leyes de la naturaleza. Detengámonos por un momento en un aspecto circular que parece anidar en las definiciones. De un lado, la delimitación fingida de cuerpos en el universo demanda reconocer el movimiento de dichos fragmentos en relación con sus vecindades; y, de otro lado, el reconocimiento del movimiento exige contar con herramientas para distinguir de sus vecinos a un fragmento del universo. La definición que hemos citado de movimiento puede reescribirse en los siguientes términos sin modificar el sentido sugerido:

Movimiento: Def la traslación de un cuerpo en relación con la vecindad de los que contactan inmediatamente con él.

La definición de cuerpo se puede, a su turno, reescribir así:

Cuerpo: ${ }_{\text {Def }}$ aquello que es trasladado a la vez con respecto a sus vecindades.

Si sustituimos la segunda definición en la primera, aceptando las modificaciones sintácticas necesarias, obtenemos la siguiente propuesta vacía:

Movimiento: ${ }_{\text {Def }}$ la traslación en relación con las vecindades de aquello que es trasladado a la vez con respecto a sus vecindades.

El círculo se puede romper o bien al proponer una definición de cuerpo que no requiera de la identificación de movimiento alguno, o bien al proponer una definición de movimiento que no requiera del reconocimiento previo de cuerpos. El primer camino, siempre que nos neguemos a admitir formas substanciales, se puede transitar si admitimos la posibilidad del vacío y reconocemos que éste es el espacio tendido entre dos cuerpos diferentes. El segundo camino se puede transitar si disponemos de un espacio absoluto que resulte independiente de los objetos que pueda alojar; es decir, un espacio en el que los objetos puedan verse como si variaran sus posiciones

\footnotetext{
${ }^{30}$ Principios (edic. francesa), AT, IX, parte II, $\S 25$, p. 76.

${ }^{31}$ Principios (edic. francesa), AT, IX, parte II, $\S 25$, p. 76, el subrayado es nuestro.
} 
relativas después de haberse reconocido sus posiciones absolutas. ${ }^{32}$ En cualquiera de los dos casos se sacrifica el espíritu minimalista de Descartes.

Daniel Garber ha tratado de mostrar que si nos restringimos a las Reglas o al Tratado del Mundo vamos a encontrar una noción relativista del movimiento que no nos permite distinguir entre movimientos reales y movimientos solo aparentes en virtud de la peculiar posición del observador. ${ }^{33}$ Garber sostiene que los Principios de la filosofía sí ofrecen un trasfondo para distinguir de manera absoluta entre reposo y movimiento sin verse obligado a postular una forma de espacio absoluto. El problema con la propuesta de Garber es que esa distinción sólo puede establecerse después de admitir la validez de las leyes de las colisiones. Ahora bien, como hemos venido tratando de defender, la inteligibilidad de dichas leyes requiere que podamos contar con criterios de individuación de cuerpos y estos, a su vez, aluden a una definición relativista de movimiento. Garber concluye: «El mundo cartesiano es un mundo de objetos geométricos que se hacen reales. Pero como él los construye, los objetos de la geometría, aun cuando ellos se dan objetivamente en la mente, estos son tomados como cosas duraderas y entonces son al menos capaces de movimiento objetivo en un tiempo objetivo». ${ }^{34}$ Ahora bien, tomar los objetos como ficciones geométricas que se hacen reales demanda criterios completos de individuación que distingan en forma clara movimiento genuino de movimiento aparente..$^{35}$

Garber no sólo advierte la circularidad entre las definiciones de movimiento y cuerpo, él también afirma que no se puede invocar el movimiento para individuar cuerpos en la cosmología cartesiana. El comentarista apela al argumento de Leibniz según el cual no tenemos criterios de identidad entre porciones del plenum cartesiano a través del tiempo, y por tanto no habría criterios para identificar el movimiento. No hay criterios claros para decir que la misma porción cambió entre dos instantes diferentes y, por tanto, que hay movimiento. Esto lleva a Garber a concluir que no se puede invocar el movimiento en general para individuar cuerpos en la cosmología cartesiana acudiendo simplemente al movimiento. También afirma, a manera de sospecha, que en el fondo Descartes no tiene licencia para hablar de cuerpos individuales. $^{36}$

Martial Gueroult, enfrentado al problema de la individuación de los cuerpos en Descartes, sugiere que la única forma de distinguir un cuerpo individual del universo compacto es apelando a la cohesión como una fuerza que tiene su origen en la actividad de Dios: «lo que hace a un cuerpo un cuerpo es la cohesión o fuerza de reposo

\footnotetext{
${ }^{32}$ También podemos valernos, como lo hace Leibniz, de espacios relativos siempre que rompamos con la pretendida identidad entre cuerpo y extensión.

${ }^{33}$ Cfr. D. Garber, Descartes' Metaphysical Physics. Chicago: The University of Chicago Press, 1992, pp. 157-172.

${ }^{34}$ D. Garber, Op. cit., p. 175.

${ }^{35}$ Garber es consciente de la circularidad que entrañan las definiciones de Descartes (Op.cit., p. 178).

${ }^{36}$ Op.cit., p. $\quad 180-181$.
} 
que pega sus partes y las hace mover en conjunto». ${ }^{37}$ Este tipo de propuestas resuelve el problema renunciando a la demanda minimalista del programa cartesiano: no introducir conceptos diferentes a extensión y sus modos (, entre ellos el movimiento).

En síntesis, la noción de cuerpo demanda una construcción ficticia del espíritu que se apoya en la posibilidad de reconocer movimiento relativo entre la porción del plenum encerrada en una superficie maximal y el plenum circundante. Sin embargo, la definición de movimiento demanda, a su turno, el reconocimiento de las ficciones elaboradas por el espíritu. No resulta fácil divisar una salida clara frente al círculo señalado. Si bien en el Tratado de la luz Descartes presenta su cosmología como una ficción sofisticada ("mundus est fabula"), hay una gran diferencia entre las dos ficciones. La fábula del mundo presenta un universo imaginario absolutamente poblado de materia extensa que, después de recibir un impulso originario dado por Dios, empieza a desarrollarse ajustado a las leyes de la naturaleza que imaginamos Dios impone imitando en el mundo su propia inmutabilidad. El genio de Descartes consiste en mostrar que un universo así fingido se parece en grado sumo al mundo real. Si el mundo así fingido cumple su tarea, podemos valernos de él para proyectar anticipaciones valiosas. Así entonces, Descartes no ofrece una explicación que da cuenta del por qué las cosas acaecen de esta u otra manera: «mi proyecto no es el de explicar [...] las cosas que existen en el verdadero mundo, sino sólo fingir uno a mi gusto, en el que nada haya que los espiritus más comunes no sean capaces de concebir y que pueda, no obstante, ser creado tal como lo habré fingido». ${ }^{38}$ La ficción que lleva al espíritu a construir cuerpos individuales no funge como una conjetura que conduce a anticipaciones acertadas; es, más bien, un criterio que introduce una convención en nuestros modos de hablar: debemos estar en condiciones de individuar objetos para a continuación referirnos a ellos en nuestro lenguaje y en las leyes o hipótesis que queramos conjeturar.

Las dos ficciones, a pesar de las diferencias, están articuladas. Dios es el creador de la materia-extensión originaria, es el garante de la conservación de dicha materia a cada nuevo instante de tiempo, ofrece el fundamento de las leyes de la naturaleza si Dios se entiende como la única causa general de todos los movimientos. Y, lo que es más importante para nuestro estudio, si bien es cierto que los cuerpos son ficciones de nuestra imaginación, estas particiones son, sub specie aeternitatis, divisiones reales: «Dios la divide verdaderamente en numerosas partes, las unas mayores, las otras menores; las unas de una figura, las otras de otra, de cualquier tipo que nos plazca fingir» ${ }^{39}$

\footnotetext{
${ }^{37}$ Cfr. M. Geroult, "The Metaphysics and Physics of Force in Descartes”, en S. Gaukroger, Descartes: Philosophy, Mathematics and Physics. Sussex: Harvester Press, 1980, p. 206p. 211-212.

${ }^{38}$ De la Luz, AT, XI, p 36.

${ }^{39}$ De la Luz, AT, XI, p 34; el subrayado es nuestro.
} 


\section{La cosmología cartesiana vista como un modelo de la Teoría M}

En un interesante estudio de metafísica, Michael Jubien (1993) ha tratado de defender que, en un cierto sentido, no existe cosa alguna, salvo si nos disponemos a reconocer que hay tantas cosas como las que nuestros esquemas conceptuales permi$\tan .^{40} \mathrm{La}$ tesis de Jubien presupone que el mundo no viene dividido naturalmente en un arreglo de cosas discretas. Así pues, ser una cosa (de hecho esencialmente separada del resto) no se define en virtud de alguna propiedad esencial o intrínseca (alguna forma substancial), sino que se define como una propiedad relacional, algo del estilo de $x$ es una cosa para nosotros en el marco de tales y tales construcciones ficticias. «Nuestra posición ontológica fundamental, explica Jubien, es que existe una suerte de material [Stuff] disperso aqui y alli y ahora y a lo largo de todo el espacio-tiempo. Parece un asunto de convención qué parcelas de tal material cuentan como cosas. La "estructura-cosa" de la distribución de tal material es algo que nosotros imponemos de una u otra forma para varios propósitos posibles». ${ }^{41}$ Salvo la diferencia que Jubien sugiere entre material y trasfondo espacio-tiempo, Descartes parece ajustarse estar ajustándose a la recomendación: hay una suerte de material-extenso [Stuff] esparcido en modos espacio-temporales, material que podría ser fragmentado o dividido por nosotros (puede ser valiéndose de superficies maximales) atendiendo a la manera como nuestros esquemas conceptuales lo demanden o lo permitan.

Jubien construye una teoría (que denomina Teoría $M$ ) a partir de una noción primitiva, dos definiciones y un axioma. La noción primitiva consiste en reconocer la posibilidad de distinguir los puntos del espacio-tiempo entre ocupados y no-ocupados. Las dos definiciones estipulan que, primero, «Una región (de espacio-tiempo) es cualquier conjunto no vacío de puntos del espacio-tiempo»y, segundo, «una región está llena si y solo si cada uno de sus puntos está ocupado»..$^{42} \mathrm{El}$ único axioma de su teoría reza así: "Si una región está llena, entonces sólo una cosa ocupa la totalidad de sus puntos». ${ }^{43} \mathrm{Si}$ admitimos la posibilidad de reconocer en la física cartesiana una estructura geométrica previa constituida por coordinaciones cuatro-dimensionales de espacio-tiempo y reconocemos, también, la noción primitiva de distinguir entre puntos ocupados y no-ocupados, podremos así intentar ver el mundo

\footnotetext{
${ }^{40}$ Adoptar esta clave de lectura nos conmina a movernos por senderos cercanos a lo que Putnam ha denominado realismo interno. Según esta perspectiva, sólo tiene sentido formular la pregunta ¿de qué objetos consta el mundo? desde el interior de una teoría o de unos instrumentos para describirla. Nelson Goodman también sostiene una posición parecida en Ways of Worldmaking; nuestros intentos por construir mundos (por describir de qué constan) sólo puede evaluarse en el marco de nuestra inclinación por hacer narraciones (ficciones).

${ }^{41}$ M. Jubien. Ontology, Modality and the Fallacy of Reference. Cambridge UK: Cambridge University Press, 1993, p. 9.

${ }^{42}$ M. Jubien. Op. cit., p. 15.

${ }^{43}$ Ibidem.
} 
cartesiano como un modelo para la teoría $M$. No tenemos que esperar que Descartes estuviese dispuesto a aceptar literalmente la noción primitiva, pues hemos visto que el filósofo no reconoce que podamos concebir una región extensa que no entrañe presencia corporal. El espacio no es, para Descartes, una entidad o estructura que exista con antelación al hecho de que haya objetos alojados allí. El espacio no es lo que queda después de que lo vaciemos de todo lo extenso-corporal. Hemos visto que para Descartes hablar de regiones espaciales no ocupadas (incluso puntos) entraña una referencia ininteligible. Esto lo podemos subsanar si exigimos que, si bien por una distinción de razón podríamos pensar en la posibilidad de admitir la existencia de puntos no-ocupados, cada uno de los puntos de la estructura geométrica previa es, de facto, un punto ocupado. ${ }^{44}$ En otras palabras, en el universo de facto de Descartes no hay puntos no-ocupados.

De lo anterior se sigue que el mundo cartesiano puede concebirse como un modelo trivial de la Teoría $M$, el modelo para el cual el conjunto completo de puntos espacio-temporales es una región llena. A juzgar por el único axioma, sólo existe una cosa en estricto sentido: el universo en su totalidad. Para decirlo de otra manera, el universo cartesiano no es una parte de otro universo más amplio que lo contiene a él como un ejemplar reducido. Así las cosas, el mundo de Descartes, visto como un modelo trivial de la Teoría $M$, coincide con la exigencia de Spinoza: no puede haber dos substancias de la misma naturaleza. Otra forma de concebir un modelo trivial de la Teoría M es imaginar un universo de coordinaciones espacio-temporales de tal manera que ninguno de sus puntos pudiese considerarse ocupado. En este nuevo universo no habría región alguna que pudiese considerarse llena (sería una inmensa nada). La Figura 1 muestra lo que podrían ser tres modelos de Teoría M. La X superpuesta en el punto de la variedad sugiere que el punto está ocupado, el círculo trazado en derredor sugiere que el punto no está ocupado.

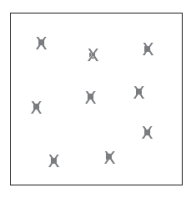

Mundo cartesiano

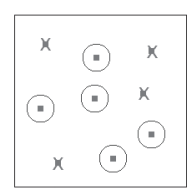

(1)

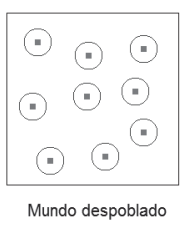

Mundo despoblado

Figura 1. Modelos de Teoría M

Imaginemos, pues, una variedad cuatro-dimensional de puntos que denominamos coordenadas espacio-temporales. Imaginemos también que de cada punto podemos decir si está o no ocupado (noción primitiva). Pensemos también que para el caso de la cosmología cartesiana admitimos que no existen puntos no-ocupados, aun cuando

\footnotetext{
${ }^{44}$ No hay duda que Descartes no estaría contento con esta sugerencia, pero de todas maneras podríamos avanzar en la construcción del modelo admitiendo esta posibilidad.
} 
concebimos la posibilidad de que pudiesen existir. Todo el universo es compacto y es, según el axioma de la Teoría $M$, la única cosa que en sentido estricto ocupa la variedad en su totalidad. Supongamos que podemos separar las coordenadas espaciales de la coordenada temporal y que contamos con algún criterio mediante el cual podamos reconocer que el punto substancial que ocupa las coordenadas $(\mathrm{x} 1, \mathrm{y} 1, \mathrm{z} 1$, t1) es el mismo punto que ocupa ahora las coordenadas. ${ }^{45}$ Esto nos obliga a agregar una noción primitiva adicional a la propuesta por Jubien; esta consiste en la habilidad para reconocer si dos puntos que se dicen ocupados en vecindades temporales, lo están por el mismo substrato o no. Llamemos a esta noción la habilidad para reconocer identidad. Podemos seguir a Descartes e imaginar que el único que puede garantizar y de hecho conocer la efectiva conservación del punto substancial en cada nueva coordenada temporal es Dios. De hecho el Dios cartesiano, en una de sus posibles interpretaciones, recrea a cada instante nuevamente el universo en su totalidad. Sostiene Descartes en Los Principios, refiriéndose a una prueba de la continua acción creadora de Dios: «Dado que sus partes [se refiere a las divisiones del tiempo] no dependen una de otra, y nunca existen simultáneamente; y en consecuencia del hecho de que nosotros existimos ahora, no se sigue que seguiremos existiendo en el siguiente instante a menos que alguna causa, en verdad la misma que nos produce desde el comienzo, continuamente nos re-cree, esto es, nos conserve». ${ }^{46}$ Martial Geroult defiende que sin el gesto continuamente creador de Dios no habría forma de darle sentido a la física cartesiana:

La existencia y duración de las cosas es debida a la presencia en ellas de una fuerza creativa que las hace existir y conserva en la existencia. De ahí es claro que mientras los modos y esencia de las cosas existentes se refieren tan sólo a extensión y movimiento, las existencias de la que se ocupa la física, las fuerzas que las animan y las leyes que las gobiernan, deben ser referidas a Dios, quien es el principio de las fuerzas por las cuales estas cosas existen, perduran y cambian. ${ }^{47}$

$\mathrm{Si}$, de un instante a otro vecino, alguna de, o todas, las coordenadas espaciales cambian aunque reconocemos la identidad substancial del punto, diremos que presenciamos un punto substancial en movimiento (o en devenir). Así las cosas, si nos damos previamente una variedad cuatro-dimensional y la posibilidad de identificar puntos substanciales que conservan su identidad con el paso del tiempo, podemos definir el movimiento sin entrar en la circularidad señalada en la sección anterior. Peter Strawson, por ejemplo, defiende que en nuestro marco conceptual reconocemos la idea de un sistema espacio-temporal de cosas materiales en el que la idea de

\footnotetext{
${ }_{45}^{45}$ Jubien no necesita introducir diferencias entre las coordenadas y reconoce la posibilidad de concebir cuerpos que constan de partes separadas temporalmente.

${ }^{46}$ Principios (edic. francesa), AT, IX, parte I, § 21, p. 34.

${ }^{47}$ Op.cit., p. 196.
} 
cada cosa material en un tiempo dado se puede relacionar de diversas maneras con ideas asociadas a otras cosas materiales. No obstante, no podríamos aferrarnos a esa idea sin una noción primitiva de identidad. Al respecto complementa Strawson: «una condición para tener dicho esquema conceptual es la aceptación incuestionable de la identidad-particular en al menos algunos casos de observación no continua». ${ }^{48}$ Sin admitir la permanencia de algo, no tendría ni siquiera sentido que nos esforzáramos en concebir leyes del mundo natural. Esta es también la exigencia que plantea Kant en la primera analogía a propósito de la constitución de la experiencia. ${ }^{49}$

Ahora podemos imaginar que estamos en condiciones de seguir la traza de un punto substancial desde sus orígenes hasta el fin de los días. Concedamos que existen esos puntos substanciales. Esta traza tiene la forma de una curva en el espacio 4-dimensional, a ella la la cual denominaremos Linea-de-mundo-del-punto-substancial. Fue Hermann Minkowski (1864-1909) quien se valió por primera vez del concepto de línea-de-mundo para presentar la cinemática propia de la Teoría de la Relatividad. Una línea-de-mundo muestra la sucesión temporal continua de posiciones espaciales asociadas con un objeto cuya identidad está garantizada; una línea-de-mundo es una curva de puntos ocupados, de los cuales asumimos que conservan su identidad substancial mientras son asociados al parámetro $t$ desde $-\infty$ hasta $+\infty$. La línea-de-mundo muestra la historia o devenir de un punto substancial (partícula) que no podría desaparecer en la nada. Si una línea-de-mundo se suspende en un momento dado, estaríamos presenciando la desaparición milagrosa de un punto substancial. Así presenta Minkowski la definición:

Nosotros fijamos nuestra atención en el punto substancial que está en el punto-mundo $x, y, z$, te imaginamos que somos capaces de reconocer este punto substancial en cualquier otro tiempo [...] Asi las cosas, nosotros obtenemos una imagen, por asi decirlo, de la carrera perdurable del punto substancial, una curva en el mundo, una línea-de-mundo, definida como aquellos puntos que pueden ser referidos univocamente al parámetro t desde $-\infty$ hasta $+\infty . .^{50}$

Previo al pasaje citado, Minkowski define punto-de-mundo como un elemento de la variedad 4-dimensional y después agrega que «Para no dejar un vacio abierto en cualquier lugar, imaginaremos que donde quiera y cuando quiera existe alli algo perceptible ${ }^{51}$. Así entonces, en el lenguaje de Jubien, cada coordenada de la variedad se puede considerar ocupada. Después complementa Minkowski, refiriéndose a ese algo que ocupa cada coordenada: «Para evitar decir "materia" o "electricidad", usaré

\footnotetext{
${ }^{48}$ P. F. Strawson, Peter F. Individuals. Londres: Routledge 1959, p. 35.

${ }^{49}$ I. Kant, Immanuel. Crítica de la razón pura. Madrid: Ediciones Alfaguara, 1993, B224.

${ }^{50}$ H. Minkowski, Hermann. "Space and Time", en H. A. Lorentz, A. Einstein, H. Minkowski \& H. Weyl, The Principle of Relativity. New York: Dover Publications, INC., 1952, pp. 75-96; p. 76.

${ }^{51}$ Ibidem.
} 
para este algo la palabra substancia $\gg .{ }^{52}$ El punto-de-mundo es un elemento de la variedad 4-dimensional, un punto-substancial es un punto de la variedad que se considera ocupado y que conserva su identidad durante toda la eternidad. Tanto en el universo de Minkowski como en el de Descartes, cada punto de la variedad está ocupado.

La Figura 2 ilustra un conjunto de coordinaciones cuatro-dimensionales, la coordenada temporal está representada en el eje horizontal y las tres coordenadas espaciales están representadas en el eje vertical. Las curvas rojas ilustran la historia de un par de puntos substanciales de los que reconocemos, en cada caso, su identidad en un intervalo de tiempo. Así entonces, podemos concebir el pleno-cartesiano-con-identidad como si estuviese poblado de puntos substanciales y sus correspondientes líneas-de-mundo. Es claro que dos líneas-de-mundo no pueden intersecarse, pues el pretendido punto de intersección tendría que ser ocupado por dos cosas diferentes, en contradicción con el único axioma de la Teoría M. Del resultado anterior también se desprende que un punto substancial no podría abrirse en dos líneas de mundo diferentes. Si al pleno cartesiano agregamos una métrica que permita atribuir velocidades a un punto substancial con respecto a otro punto que repose en el origen del sistema de coordenadas, es decir, con respecto a un punto substancial cuya línea de mundo coincida con el eje temporal (Figura 2), podemos decir que dos líneas-de-mundo son paralelas, en un instante definido, si las velocidades asociadas a los puntos substanciales en dicho instante coinciden, y son paralelas en un intervalo de tiempo si los son en cada uno de los instantes que pertenecen al intervalo (las dos líneas-de-mundo de la Figura 2 representan, con cierta aproximación, líneas de mundo paralelas). Si quisiéramos agregar restricciones relativistas, tendríamos que exigir que ninguna velocidad supere cierto valor límite (la velocidad atribuida a la luz). Ahora bien, podemos exigir que el punto substancial que reposa con respecto al sistema de referencia acoja la presencia del observador cartesiano. Así las cosas, no necesitamos postular un espacio absoluto abstracto para establecer movimientos "reales", pues basta con erigir el observador cartesiano como el punto de referencia absoluto. La línea-de-mundo del observador cartesiano es la referencia con respecto a la cual decimos si otro punto-substancial se mueve o reposa.

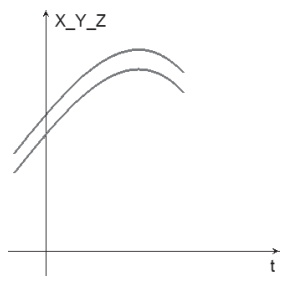

Figura . Lineas-de-mundo-de-puntos-substanciales (líneas paralelas)

\footnotetext{
${ }^{52}$ Ibidem.
} 
En síntesis, nos damos una variedad 4-dimensional, distinguimos las coordenadas espaciales de las temporales, reconocemos que cada uno de los puntos de la variedad se encuentra ocupado por un punto substancial cuya identidad podemos reconocer desde el momento de la creación hasta el final de los días y, en consecuencia, podemos seguir la traza de dicho punto substancial en una curva continua que denominamos línea-de-mundo. Suponemos también que podemos introducir una métrica y relaciones topológicas de vecindad para poder adscribir velocidades a los puntos substanciales en instantes definidos. A cada uno de estos puntos substanciales le cae bien la descripción que hemos citado de Descartes en el sentido de que cada uno de ellos es una cosa (substancia en el sentido de Minkowski) que permanece «quatenus est simplex et indivisa». Strawson, aunque no se vale del lenguaje de las líneas-de-mundo, describe así las tesis que atribuye a ciertos filósofos (sin indicar quiénes son):

Al razonar así, puede decirse que ellos [los filósofos a los que alude] llaman la atención acerca de la posibilidad de reconocer una categoría de objetos que nosotros en efecto no reconocemos: una categoría de objetos cuatro-dimensionales, los cuales podrían ser llamados "cosas-en-proceso", y de los cuales cada una de las partes temporalmente sucesiva, que es tres-dimensional, es, como si fuera, la cosa tomada en los estadios sucesivos de su historia desde el comienzo hasta el final. ${ }^{53}$

Nos preguntamos ahora: ¿cómo introducir particiones en el mundo cartesiano para construir cuerpos en el sentido laxo? Podemos valernos de las líneas-de-mundo, las relaciones topológicas de vecindad y la métrica para crear las particiones que habríamos de denominar cuerpos. Proponemos la siguiente definición: "dado un instante de tiempo $t$ y un modelo cartesiano de la teoría $M$, una región de puntos espaciales topológicamente conexa ${ }^{54}$ se dice una partición cartesiana o un cuerpo si y sólo si (i) las líneas-de-mundo de los puntos de dicha región ${ }^{55}$ son paralelas en ese instante y, además, (ii) la superficie que envuelve dicha región es tal que ninguna de las líneas-de-mundo de los puntos substanciales en dicha superficie es paralela con las líneas de mundo de los puntos de la región encerrada". El primer componente trata de asegurar que los puntos substanciales encerrados en la partición carecen de movimiento relativo entre sí, en tanto que el segundo advierte que los puntos substanciales de la partición se mueven en relación con la frontera de la región. La frontera es, entonces, una superficie maximal o el lugar externo del cuerpo definido. La Figura 3 ilustra las intuiciones básicas de la propuesta. La Figura, por razones de

\footnotetext{
${ }^{53}$ P. Strawson. Op. cit., pp. 56-57.

${ }^{54}$ Intuitivamente, un conjunto conexo es aquel formado por una sola pieza y, en consecuencia, no se puede dividir.

${ }^{55}$ Sobra indicar que debe tratarse de una región llena pues en el modelo cartesiano cualquier región se considera llena.
} 
comodidad en la representación, muestra el caso en una variedad tri-dimensional. La recta horizontal muestra el devenir del marco de referencia. Una línea de mundo recorre dicho eje (el devenir del observador cartesiano). Las cinco líneas-de-mundo (entre las cuales obviamos la exigencia de conexidad) muestran la historia de cinco puntos substanciales independientes. El sector transversal trazado identifica una partición en el continuo, partición que podemos identificar con un cuerpo. En este caso, debido a que la variedad es 3-dimensional y no 4-dimensional, la partición no se construye a partir de una superficie maximal, sino de una línea-poligonal-maximal. Las tres líneas centrales indican que justo en el cruce no se presenta movimiento relativo entre los componentes. Mientras que las dos líneas en las vecindades del sector ilustran que tales entidades cuentan con movimiento relativo con respecto a la unidad encerrada. De otro lado, la unidad corporal señalada por el sector no se conserva en el tiempo; unos instantes después, las tres líneas de mundo centrales ilustran la aparición de movimiento relativo entre ellas. La definición sugiere un criterio para construir un cuerpo que conserva la unidad estructural tanto como dure el intervalo en el cual las líneas de mundo conserven el paralelismo aludido. Esta circunstancia de la construcción puede verse en conflicto con el sentido común y el uso acostumbrado del término cuerpo, pues en el lenguaje corriente reservamos este término para unidades que se conservan en periodos considerables de tiempo (incluso siglos cuando se trata de montañas, por ejemplo).

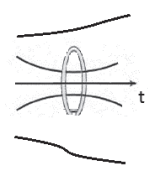

Figura . Particiones en el continuo cartesiano

Así las cosas, un cuerpo puede odría definirse como un manojo-de-líneas-de-mundo concebido de tal forma que dichas líneas son paralelas mientras atraviesan una región de la variedad. En el peor de los casos, es decir, cuando existe tal agitación en el mundo que no resulta factible hallar dos líneas-de-mundo de puntos conexos que sean paralelas, se puede sostener que cada línea-de-mundo es, de suyo, un cuerpo independiente.

Nicolás Malebranche, cuidadoso estudioso y a la vez crítico estudioso de Descartes, advirtió con razón en su Recherche de la vérité:

Si Dios al crear el mundo hubiera producido una materia infinitamente extensa sin imprimirle ningún movimiento, todos los cuerpos no habrían sido diferentes unos de otros. Todo el mundo visible no sería todavía en el presente más que una masa de materia o extensión que bien podría servir para hacer conocer la grandeza y la 
potencia de su Autor; pero no habría esa sucesión de formas y esa variedad de cuerpos que constituyen toda la belleza del universo y que llevan a todos los espíritus a admirar la sabiduría del que lo gobierna. (RV, pp. 469-470). ${ }^{56}$

Hay en la física de Descartes una permanente tensión entre el continuo (plenum) y lo discreto (los objetos reconocidos como tal). De un lado la metafísica le obliga a advertir, en forma al parecer clara y distinta, la existencia de una única substancia extensa sin hiato alguno en su constitución y naturaleza. En otras palabras, la razón le impone con fuerza un universo pleno. De otro lado, los problemas inmediatos de la ciencia natural le obligan a hablar de objetos perfectamente individuados, objetos que en un cierto sentido se podrían separar del plenum del universo. No es fácil renunciar a alguno de los dos polos y tampoco es simple conciliarlos.

No hay cuerpos per se en el mundo. Los cuerpos no nos son dados ab initio. Ellos no son el ingrediente básico de nuestras construcciones. Hay una masa extensa indefinida y sin intersticios. Dios pudo provocar en algún momento algún desbalance (movimiento) en algún sector e imponer unas leyes de conservación que habrían de regular el intercambio entre diferentes particiones de dicho universo. Así, con el temblor original de Dios se dio inicio a una rapsodia de alteraciones entre las potenciales particiones del continuo. Cuando un espíritu, atento a las normas de construcción conceptual previstas por una especie de razón universal, procede a realizar particiones ficticias e historias de dichas particiones atadas a las leyes de conservación que él conjetura que Dios ha puesto en el mundo, se verá haciendo narraciones extraordinarias de unidades que per se carecen de existencia independiente. Entre tanto, la masa entera del universo permanece incólume, como si a ella nada le hubiese pasado en absoluto. La historia del mundo es, pues, una ficción nuestra atada a las pretendidas leyes de conservación impuestas a las particiones que construimos en el mundo. En otras palabras, narramos qué aspecto tendrían las particiones que de manera ficticia creamos en el continuo si damos por sentadas las regularidades que manifiestan la acción conservadora de Dios. Nosotros, que jugamos a diseñar narraciones consistentes, nos damos variedades 4-dimensionales e imaginamos una danza frenética de líneas-de-mundo entre las cuales, para auxiliarnos en nuestras descripciones, de vez en cuando hallamos manojos-de-líneas-de-mundo que tenemos por cuerpos.

\footnotetext{
${ }^{56}$ N. Malebranche. Recherche de la verité. Paris: Galerie de la Sorbonne, 1991, pp. 469-470. Malebranche procede después por analogía, y sugiere que las inclinaciones son al mundo espiritual lo que el movimiento es al mundo material. En ese orden de ideas, son las inclinaciones las que determinan los criterios de individuación de los espíritus así como es el movimiento el que permite hablar de un cuerpo a diferencia de otro.
} 


\section{Complementos finales}

Hemos mostrado que la tensión que existe entre un universo compacto y la referencia a cuerpos particulares en las leyes de la naturaleza se puede salvar si admitimos que la noción de cuerpo individual alude a una construcción ficticia adelantada por el espíritu para encerrar en superficies maximales porciones del material original que cumplen con dos condiciones: (i) ellas se encuentran en reposo relativo entre sí y (ii) ellas se encuentran en movimiento relativo con respecto a las porciones aledañas. Hemos mostrado que la cosmología cartesiana puede presentarse como un modelo trivial de la Teoría $M$ de Michael Jubien. También hemos agregado una noción primitiva a la ontología de Jubien para garantizar el reconocimiento de identidad. Así las cosas, nos ayudamos con la noción de línea-de-mundo en una variedad 4-dimensional y nos valemos de relaciones topológicas de vecindad y una métrica para reformular la definición de cuerpo como una partición del continuo cartesiano. Ahora mencionaremos brevemente tres consecuencias importantes que se derivan de esta particular aproximación a la cosmología cartesiana: evidencias empíricas, individuación de cuerpos en el campo visual e individuación de cuerpos vivos.

(i) Evidencias empíricas. Si los cuerpos particulares a los que alude la tercera ley de la naturaleza (conservación de la cantidad de movimiento en choques simples) no se ajustan a la materia encerrada en una superficie maximal, no sería posible obtener buena evidencia empírica en favor de dicha ley. La razón es sencilla. La conservación de la cantidad de movimiento presupuesta en dicha ley asume, si acogemos los términos que hoy en día usamos al respecto, que los choques son elásticos. Esto es, hay buena evidencia empírica para la tercera ley si se asume que no existe intercambio de energía que se consuma en forma de agitación interna de las partes constitutivas de los cuerpos. Los choques entre bolas de billar muestran evidencias en favor de la ley siempre que podamos imaginar que dichas bolas no aumentan su temperatura (agitación interior) durante las colisiones. En verdad, es casi imposible encontrar particiones en el mundo físico que cumplan con exactitud las condiciones impuestas para la construcción de cuerpos. En cada uno de los objetos que tenemos por cuerpos físicos existe una dinámica muy rica de agitaciones internas. Sin embargo, para efectos prácticos en muchas ocasiones podemos ignorar la dinámica interior y comportarnos como si cada una de las partes que pudiésemos concebir en un cuerpo reconocido como tal se desplazara de la misma manera que lo hace el conjunto. ${ }^{57}$ Esto explica por qué Descartes agrega una aclaración en la definición que él

\footnotetext{
${ }^{57}$ Por eso en ocasiones a los físicos les resulta útil referirse, más bien, al centro de masa de un sistema en lugar de pretender seguir la historia particular de cada uno de sus componentes. Garber presenta así el asunto: «lo que Descartes tiene en mente por un movimiento común es el movimiento de un centro de gravedad de las partículas que conforman el cuerpo en cuestión» (Op. cit., p. 178)
} 
propone de cuerpo (y que nosotros citamos atrás e ignoramos en eseel momento): « [...] todo lo que es transportado a la vez, aunque esté compuesto de partes diversas que emplean su agitación para producir otros movimientos». ${ }^{58}$

(ii) Individuación de cuerpos en el campo visual. Si la individuación de cuerpos, en el horizonte que provee el campo visual, es también una ficción del espíritu, podemos hemos de concluir que los objetos separados que se perciben en dicho horizonte no son dados per se e independientes de nuestras determinaciones. Antes al contrario, es el espíritu quienel que, con sus construcciones y determinaciones ficticias, delimita los objetos allí reconocidos. Es como si del ojo emanaran unos rayos noéticos que determinan sobre qué aspectos del horizonte perceptual ha decidido el alma fijar su atención..$^{59} \mathrm{El}$ contraste entre el color en los bordes de un objeto construido como ficción nuestra y los colores del trasfondo de horizonte de nuestro campo visual pueden dar la pauta para la individuación óptica. En efecto, la diferencia de colores en la cosmología cartesiana alude a las diferencias de velocidad angular asociadas con los vórtices que acompañan la presión centrífuga que el medio ejerce sobre el fondo de la retina. ${ }^{60}$ En ese orden de ideas, el contorno de un objeto divisado en el campo visual obedece a los contrastes en las líneas-de-mundo en el límite que define los bordes de un objeto visual. El cambio de color en los límites de un objeto contemplado en el horizonte de contraste que ofrece el campo visual es un síntoma que le permite al observador cartesiano reconocer con prontitud los bordes de una superficie maximal, o mejor, de una línea-poligonal-maximal, toda vez que el espacio del campo visual es bi-dimensional.

(iii) Individuación de cuerpos vivos. Dadas las dificultades que hemos señalado en relación con la individuación de los cuerpos físicos en un universo que no admite intersticios vacíos, también es legítimo que preguntemos: ¿qué permite individuar ahora un cuerpo físico como un cuerpo con vida o un cuerpo animado? No en vano Strawson plantea que las dos construcciones básicas de nuestro esquema conceptual son las construcciones de cuerpos, por un lado, y personas, por el otro. ¿Puede el cuerpo de un ser humano o el cuerpo de un animal concebirse a la manera de la porción de materia universal encerrada en una superficie maximal? La respuesta categórica es “¡No!”. Como ha sugerido Descartes, en el interior del cuerpo de un ser humano existe una muy compleja dinámica y rapsodia de actividad interna: la sangre circula animada por los impulsos que le da el corazón quien es un almacén de

\footnotetext{
${ }^{58}$ Principios (edic. francesa), AT, IX, parte II, $\S 25$, p. 76.

${ }^{59}$ Hemos tomado la noción de rayo noético de H. Putnam (Razón, verdad e historia. Madrid: Editorial Tecnos, 1988, p. 60) quien la usaba para ilustrar una versión de lo que él denominaba una teoría mágica de la referencia. Según esta versión, habría que suponer la existencia de los rayos noéticos para concebir la conexión entre palabras y símbolos mentales con sus pretendidos referentes.

${ }^{60}$ Cfr. Dióptrica, $A T$, VI, p. 131; Meteoros, AT, VI, pp. 325-344; Del hombre, AT, XI, pp. 174-177.
} 
fuego sin luz, una cantidad inmensa de espíritus animales circula por los conductos nerviosos, los filamentos encerrados en los nervios reaccionan a las presiones que reciben las superficies de irritación (sentidos), los pulmones se dilatan para introducir aire que habría de condensar la sangre que viene del corazón, los alimentos ingresan al sistema digestivo e insuflan los nutrientes que navegan en la sangre. En virtud de toda esa inevitable agitación interna, nos vemos obligados a buscar otro tipo de unidad para el cuerpo asignado a un ser animado. El cuerpo de un ser animado no es la masa del universo encerrado en una superficie maximal.

Descartes concibe el cuerpo humano como una máquina que articula diferentes cuerpos físicos al servicio de una función integral: la particular conservación de la unidad cuerpo-alma. Se trata, más bien, de un complejo mecanismo cuyas partes se articulan armónicamente para llevar a cabo tareas o funciones bien establecidas. Organismo es, quizá, un término más adecuado para referirse a la porción de materia encerrada en un cuerpo animado. No hay en la obra de Descartes, o por lo menos no lo hemos encontrado, un intento por perfilar con claridad los límites precisos de aquello que podría encerrar un organismo. En principio, y en coherencia con lo anotado en el artículo 30 de De las pasiones ${ }^{61}$ podemos pensar que un organismo encierra todo aquello que pueda estar bajo el control o el escrutinio de un alma (mente). En una carta al padre Mesland (9 de febrero de 1645) explica Descartes que la noción de cuerpo referida a un organismo vivo (un humano, por ejemplo) debe ser más compleja: «cuando hablamos del cuerpo de un hombre, no entendemos una parte determinada de la materia [...] solamente entendemos toda la materia que está unida y ensamblada con el alma de ese hombre; de suerte que bien sea que esta materia cambie y que su cantidad aumente o disminuya, nosotros creemos siempre que es el mismo cuerpo, ídem numero, siempre que permanezca junto y unidos sustancialmente a la misma alma $\rangle^{62}$

La particular conexión del cuerpo y el alma es advertida también en la sexta meditación, aunque luego se desecha como una opción legítima para probar la existencia del mundo exterior. Dice Descartes:

No faltaba tampoco razón para que yo creyera que este cuerpo (al que con un cierto derecho particular llamaba mío) me perteneciera con más propiedad [...] Porque, en efecto, nunca podía ser separado de él como de los demás cuerpos; sentía en él $y$ por él todos mis apetitos y todas mis afecciones; $y$, en fin, yo era afectado por sentimientos de placer y dolor en sus partes, y no en las de otros cuerpos que estaban separados de él. ${ }^{63}$

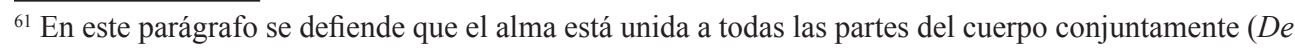
las pasiones, AT, p. 351).

${ }^{62}$ AT, IV, p. 166.

${ }^{63}$ Meditaciones, $A T$, IX, medit. VI, p. 60.
} 
Están bajo el escrutinio de un alma todas aquellas porciones a las que llegan terminales nnerviosas que se han originado previamente en las vecindades del centro del cerebro, allí donde se encuentra la glándula pineal. Así entonces, forma parte del cuerpo de un ser humano animado (complejo) cada una de las superficies maximales que encierran porciones de la materia universal a la que llegan, sin pérdida de continuidad, terminales nerviosas que convergen en el centro de un cerebro. En ese orden de ideas, cuando nos es guillotinado un dedo, dejamos de considerarlo parte de nuestro cuerpo (organismo), porque habríamos roto el posible control o escrutinio que podría ejercer nuestra alma sobre esa desafortunada porción de materia universal. Tirado el dedo en el piso, sin conexión con nuestras terminales nerviosas, ya no nos interesa su suerte. No tenemos el mismo sentimiento de pérdida cuando nos despojamos de nuestro cabello pues, salvo en la unión con el cuero cabelludo, no llegan terminales nerviosas a los fragmentos que constituyen un hilito del pelo. ${ }^{64} \mathrm{Un}$ organismo podría llegar a entenderse como un manojo de líneas-de-mundo vecinas espacialmente, que, para efectos prácticos, podemos considerar paralelas y que son llevadas al unísono de aquí para allá de acuerdo a las determinaciones o intenciones de un alma.

\section{Referencias bibliográficas}

ARISTÓTELES. (1998). Física. Madrid: Editorial Gredos.

DESCARTES, René. (1897-1910). Oeuvres de Descartes. Paris: Léopold Cerf (editor); publicadas por Charles Adam \& Paul Tannery con el auspicio del Ministerio de Instrucción Pública; 12 volúmenes.

GARBER, Daniel. (1992). Descartes' Metaphysical Physics. Chicago: The University of Chicago Press.

GAUKROGER, Stephen. (1980). "Descartes' Project for a Mathematical Physics", en S. Gaukroger (editor), Descartes: Philosophy, Mathematics and Physics. Sussex: The Harvester Press Ltd, 1980, pp. 97-140.

GEROULT, Martial. (1980). "The Metaphysycs and Physics of Force in Descartes", en S. Gaukroger (editor), Descartes: Philosophy, Mathematics and Physics. Sussex: Harvester Press Ltd., p.p. 196-229.

\footnotetext{
${ }^{64}$ Anota Malebranche en la Recherche de la Vérité: «Es a causa del instinto del sentimiento por lo que estoy persuadido de que mi alma se halla unida a mi cuerpo o de que mi cuerpo forma parte de mi ser; no tengo evidencia de ello, tampoco lo conozco por la luz de la razón: es por el dolor o por el placer que siento cuando los objetos me afectan. Se nos pincha en la mano y sufrimos por ello; nuestra mano, entonces, forma parte de nosotros mismos. Se desgarra nuestro traje y no sufrimos nada por ello; nuestro traje, pues, no está en nosotros mismos. Se nos cortan los cabellos sin dolor, nos los arrancan con dolor, esto pone en un aprieto a los filósofos, y no saben qué decidir» (op. cit., pp. 645-646). Entre los autores persisten diferencias importantes en relación con este punto.
} 
GOODMAN, Nelson. (1978). Ways of Worldmaking. Indianapolis: Hackett Publishing Company.

JUBIEN, Michael (1993). Ontology, Modality and the Fallacy of Reference. Cambridge UK: Cambridge University Press.

KANT, Immanuel. (1993). Crítica de la razón pura. Madrid: Ediciones Alfaguara.

LEWIS, G. (1950). L'individualité selon Descartes. Paris: J. Vrin

MALEBRANCHE, Nicolás. (1991). Recherche de la verité. Paris: Galerie de la Sorbonne.

MINKOWSKI, Hermann (1952). "Space and Time", en H. A. Lorentz, A. Einstein, H. Minkowski \& H. Weyl, The Principle of Relativity. New York: Dover Publications, INC., pp. 75-96.

PUTNAM, Hilary. (1988). Razón, verdad e historia. Madrid: Editorial Tecnos. Traducción de José Miguel Esteban Cloquell.

SPINOZA, Baruch. (1980). Ética demostrada según el orden geométrico. Barcelona: Ediciones Orbis.

STRAWSON, Peter F. (1959). Individuals. Londres: Routledge.

Carlos Alberto Cardona Sú́rez

Profesor Titular de la Universidad del Rosario (Bogotá-Colombia)

Escuela de Ciencias Humanas

carlos.cardona@urosario.edu.co

Juan Raúl LOAIZA Arias

Auxiliar de investigación de la Universidad del Rosario

(Bogotá-Colombia)

Estudiante de maestría de la Humboldt-Universität

zu Berlin (Alemania)

Escuela de Ciencias Humanas / Berlin School of Mind and Brain

loaiza.juan@urosario.edu.co 